\title{
Design of new daylight simulators on architectural scale models
}

\author{
Thierry Blandet ${ }^{2}$, Levin Erbilgin ${ }^{1, *}$, Nicolas Vergnes $^{2}$, Nicolas Hoerter ${ }^{2}$, and Olivier \\ Lehmann $^{1}$ \\ ${ }^{1}$ National Strasbourg School of Architecture, Research Department, 67068 Strasbourg Cedex, France \\ ${ }^{2}$ Telecom Physique Strasbourg, ICube laboratory (IGG team), Strasbourg University, France
}

\begin{abstract}
To enable students to evaluate the impact of light on the scale models of their architectural projects, two physical simulation systems have been built: a mirror box and a sun and sky simulator. Both devices integrate LED-based light sources. We have integrated several models for the computation of the sky image: luminance models and colour appearance models. The integration of the recent and evolving LED technologies explains how our simulators are innovative.
\end{abstract}

\section{Introduction}

At the School of Architecture of Strasbourg (ENSAS), teachers developed a lighting simulation activity with the objective of placing the students under experimental conditions and considering the issues of light ambiences $[1,2]$. This is a way to directly enter into the architectural space and its conception by integrating physical and sensory phenomena. The teaching is based on the use of scale models under controlled light conditions and computer simulations.

Until now, experiments were conducted at the Belgian Building Research Institute (BBRI) in Louvain-la-Neuve, in collaboration with the Climat \& Architecture laboratory of the Louvain-la-Neuve School of Architecture [3]. This pedagogical agreement allowed teachers to define their educational needs and to gain experience with the use of these simulators. The teaching feedback and the relevant exchanges between BBRI and ENSAS technical teams led to the definition of the design and the technology involved in our simulators.

The Strasbourg School of Architecture decided to take advantage of a recent building extension to develop its own laboratory made by two high-tech devices designed for experimentation, research and teaching purposes: a mirror box and an artificial sun and sky. Both are installed on the ground floor of one of the two buildings that make up ENSAS. This educational and academic initiative required the development of modular systems to ensure the optimization and maintenance of the simulators. We will detail the design and the main

\footnotetext{
*Thierry Blandet: thierry.blandet@unistra.fr; Levin Erbilgin: levin.erbilgin@strasbourg.archi.fr
} 
characteristics of the sun \& sky simulator by presenting the several prototypes required for its conception.

\section{Theoretical approach}

\subsection{State of the art of daylight simulators}

Daylight simulators applied to architecture models have been developed since the middle of the twentieth century. Each laboratory develops its own simulators according to the current technologies and depending on the requirements expected on the results $[4,5,6]$.

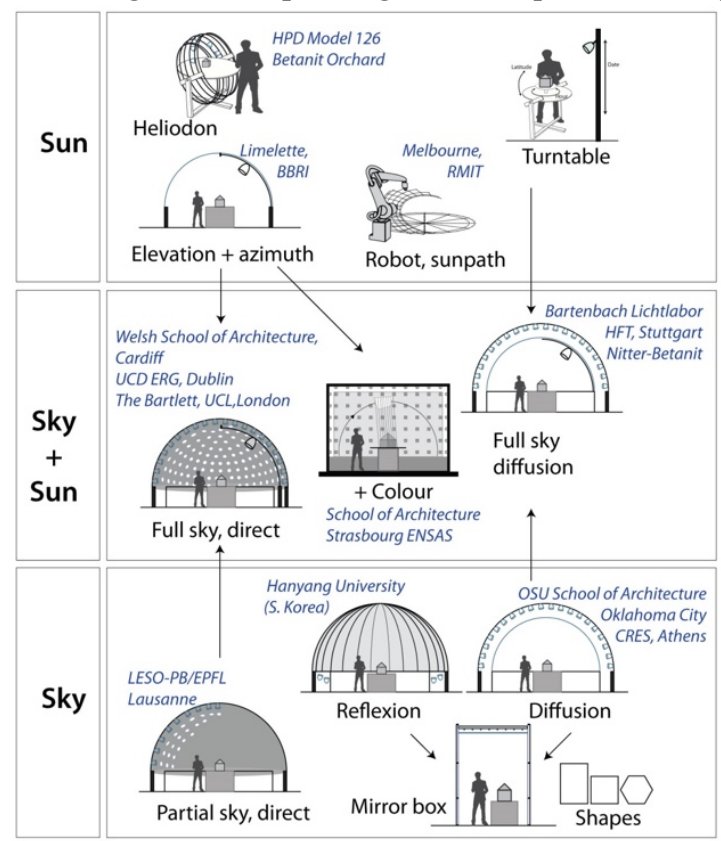

- The figure on the left presents all the daylight simulators by organizing them in three groups depending on the lighting emission they reproduce (only the sunlight, only the light emitted by the sky or the light emitted by both the sun and the sky).

- An artificial sun reproduces the sun path defined by a sequence of azimuth and elevation values. This path is relative to the model, so the artificial sun can move and illuminate the model (heliodon), the model can rotate under a static light source, or the sun handles the elevation while the model consider the azimuth. The mechanical movement is mostly linear even if a 6axis robot can also reproduce the sun path [7]. The sun can be simulated using a spotlight or a parabola to produce a parallel beam.

Fig. 1. Daylight simulators, a state of the art

The colour of emission of an artificial sun is usually white, and the intensity is possibly dimmable depending on the period of the day simulated.
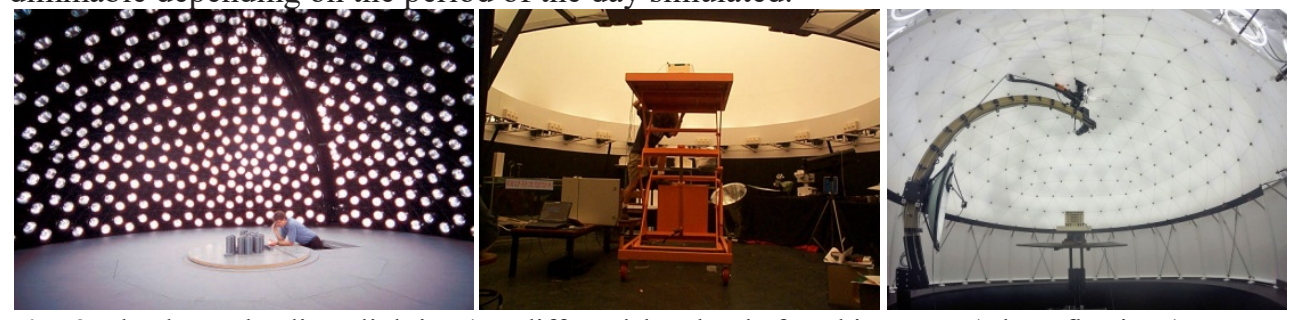

Fig. 2. Sky domes by direct lighting (Cardiff, Welsh School of Architecture $\odot$ ), by reflection (Hanyang University, South Korea $\odot$ ) and diffusion (HFT, Stuttgart $($ )).

The sky vault can be partially [8] or fully reproduced. There are three ways to reproduce sky light: by direct lighting, by reflection, or by diffusion. By reflection, spotlights disposed below the model illuminate a white dome, the reflections on the dome illuminate the model. 
Considering that the real sky emits a diffuse light, the most realistic principle is a sky working by diffusion. Light sources are disposed behind a diffusing material. Usually artificial skies have spherical shapes [9]. The most realistic systems combine an artificial sky and a mechanical sun.

\section{Experimentation, study, research and prototypes}

Considering the state of the art, we decided to develop a simulator that combines an artificial sky working by diffusion and an artificial sun. Regarding the current lighting technologies, we decided to work only with LED sources which allow the control of the luminous flux and the colour with an excellent energetic performance. For the artificial sky, we chose RGB sources individually controllable and high-power RGBW sources for the sun.

To obtain a Lambertian diffusion and reach a realistic sky, it is necessary to find the optimal diffusing material and the right distance between the light sources and this material. Because LED technology offers a possible control of each source, it is no longer necessary to build the sky with a dome shape, therefore we chose a cubic shape. After calibration, each source will have the same contribution to the centre of the experimental platform.

\subsection{Diffusing panels scattering characterization}

\subsubsection{Experimental setup}

To characterize the diffusion of a material, it is necessary to determine its transmission factor depending on the angle of observation. To achieve these measurements, a directional source is more appropriate than an extended source. The source used is a He-Ne continuous laser.
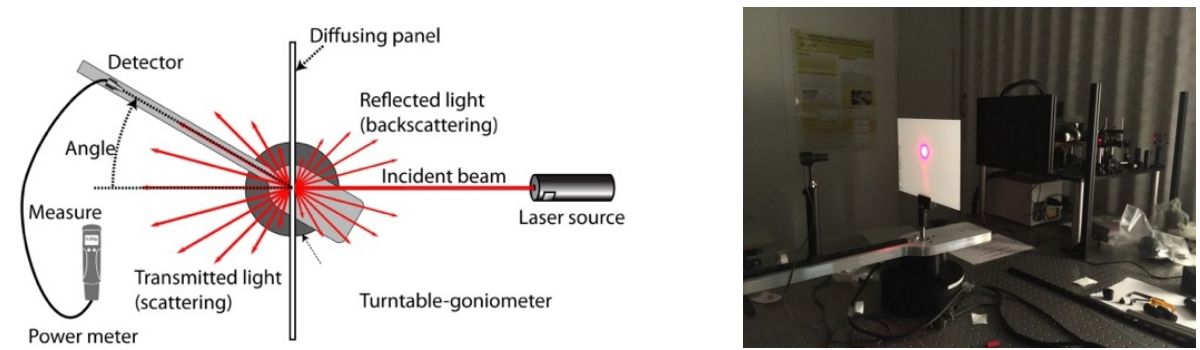

Fig. 3. Experimental set-up for scattering characterization

The laser beam is perpendicular to the sample, and a power meter is on a computer controlled mobile arm. Scattering is a balance between light transmission at $0^{\circ}$ and transmission for higher angle values. To limit the light losses, the selected material should not be too absorbent. Theoretically a perfect diffuser commonly qualified as Lambertian is a material through which its illuminance is angularly uniform. The intensity value I depending on the angle of observation $\theta$ for a Lambertian diffuser is defined by the following equation:

$$
I=I_{0} \cos \theta
$$

The objective of these measures is to identify which sample (fire resistant) is the closest to the luminous intensity defined by equation (1). 


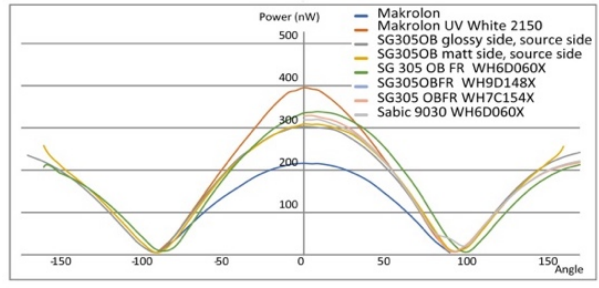

Fig. 4. Samples scattering characterization

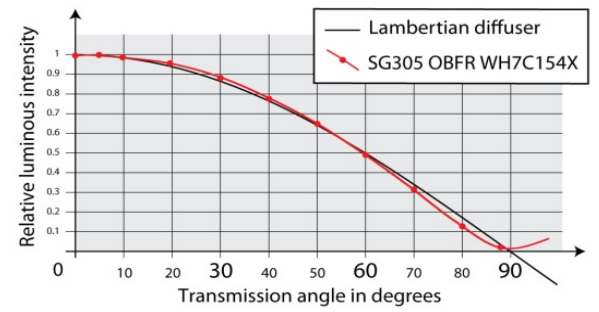

Fig. 5. Lambertian diffuser vs SG305OB

Finally, the results led to select the SG3050B FR Lexan polycarbonate diffusing panel with colour reference $\mathrm{WH} 7 \mathrm{C} 154 \mathrm{X}$.

\subsubsection{Diffusing panel junction design}

The standard dimension of the selected product is $3.05 \mathrm{~m} \times 2.05 \mathrm{~m}$. To cover a large surface several diffusing panels are arranged next to each other.

To avoid "light leaks" the following junction has been designed. The two millimetres gap between each panel allows to take into account the polycarbonate thermal dilatation and the tolerance required by the structure.

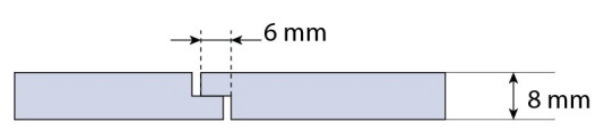

Fig. 6. Junction between two diffusing panels

\subsection{Sky prototype: presentation \& details}

\subsubsection{LEDs distribution and distance between sources and diffusing panels}

The purpose is now to define the LEDs disposition on the wall of the artificial sky, the optimal distance between the sources and the diffusing panel.
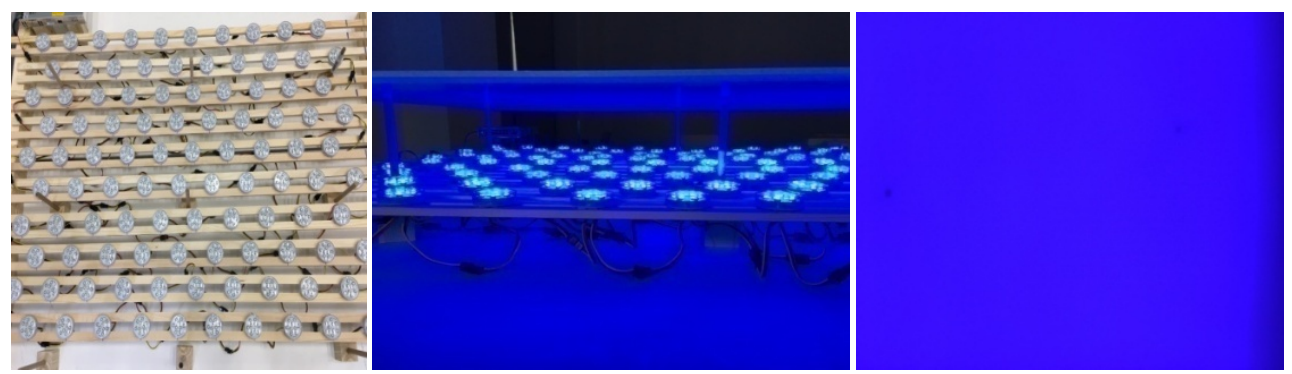

Fig. 7. Artificial sky prototype in a staggered arrangement and visual impact validation

One-hundred-pixel balls lights are disposed in a staggered arrangement. The $10 \mathrm{~cm}$ distance between the sources and the diffuser permits to obtain a homogenous scattering. The sources used in this prototype are screwed on the back and their dome is not necessary. Therefore, LED sources have been specially manufactured to meet our specifications (12 RGB LED sources on a $67 \mathrm{~mm}$ flat square on an aluminum plate drivable through the WS2811 protocol). 


\subsubsection{Pixel mapping}

In the simulator, each LED source composing the sky is individually controlled via a pixel mapping process. To fit with our specific needs, we decided to develop our own LED driving system using Arduino devices.

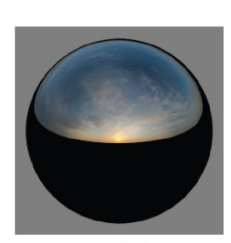

Image of the sky
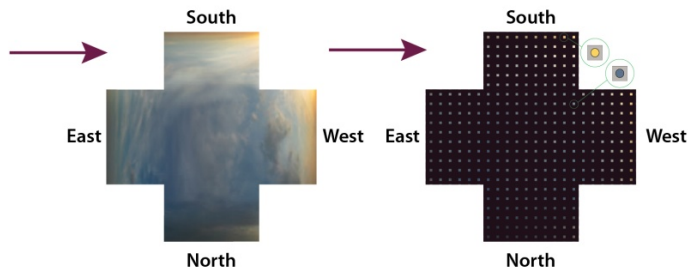

Fig. 8. Pixel mapping principle

We developed software able to compute the images of the sky, then, knowing the position and the address of each source, it is possible to consider our artificial sky as the display system for these images. To validate our approach, the final structure of the artificial sky was reproduced on a small-scale prototype $(580 \times 580 \times 460 \mathrm{~mm})$. We disposed in a staggered arrangement one hundred RGB light sources inside the model.
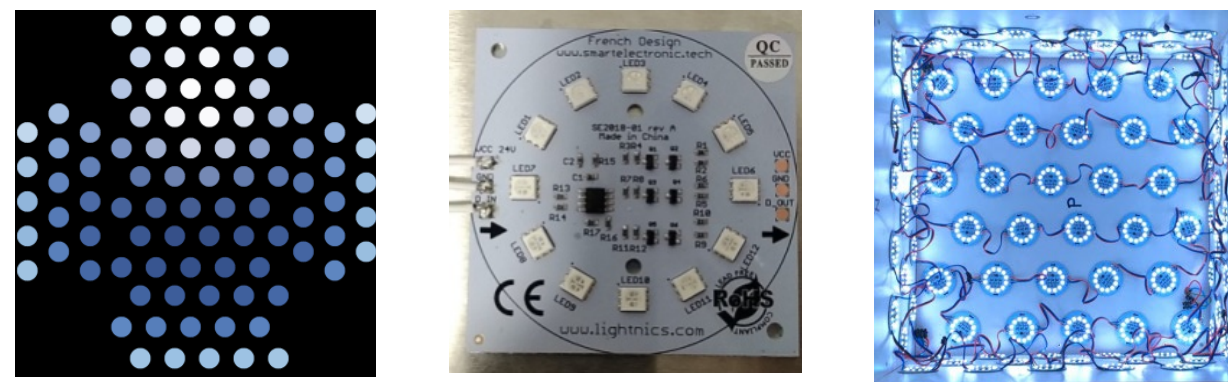

Fig. 9. The pixel mapping in our software, our specific LED source and the front view of the prototype

In addition to the pixel mapping, this prototype served as a support to control a fish-eye camera that will be needed for the final sky calibration and the visual feedback of the inside of the studied architectural model.

Because of the cubic shape, the calibration of the final system consists of assigning to each LED the right intensity so that each one has the same contribution to the centre of the experimental platform.

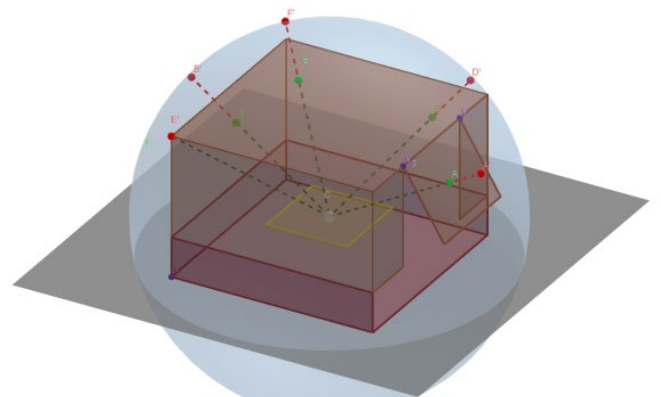

Fig. 10. Theoretical position of each LED source for calibration process 


\section{Our sun and sky simulator}

\subsection{General presentation}

In our system, the model is placed on a circular technical platform. Our artificial sky has a cubic shape with the following inside dimensions: $4780 \mathrm{~mm}$ x $4780 \mathrm{~mm}$ x $3780 \mathrm{~mm}$ (width $\mathrm{x}$ length $\mathrm{x}$ height). The user defines the geographical position of the desired solar path as well as the day. During a typical three minutes simulation, the sun goes from sunrise to sunset, follows its trajectory in the sky [10], the colour and intensity of the artificial sun and sky will evolve in the simulated day as in reality. The sun and sky simulator has to be considered mainly as a qualitative device.

The experiments can be observed directly by the users from the outside of the simulator through a large opening at north. A bright awning compensates the loss of light due to this opening. The simulation is recorded by cameras positioned inside and around the architectural model.
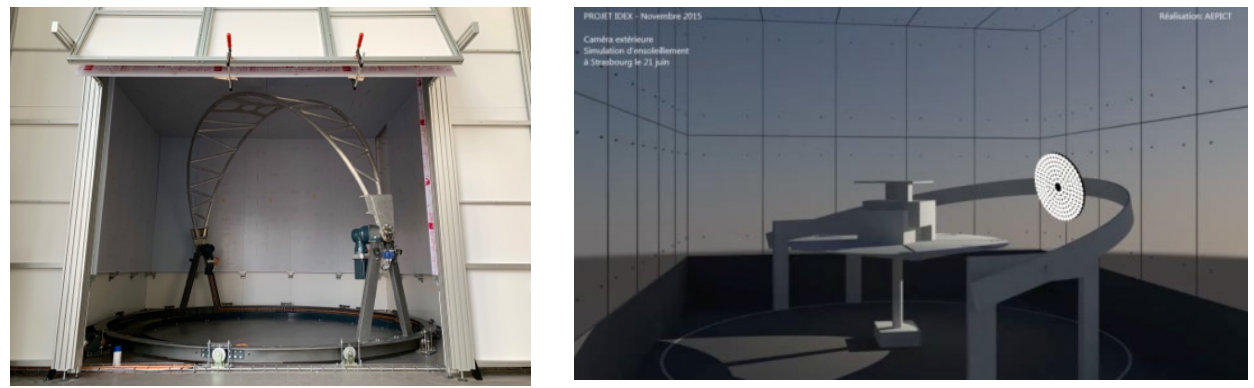

Fig. 11. Mechanical sun within the artificial sky and a computed simulation of a model within the cubic shape of our sun-sky simulator

8,000 RGB LED sources are distributed on the walls and ceiling of the structure behind polycarbonate $8 \mathrm{~mm}$ thick diffusing panels. Our software calculates in real-time the image of the sky, through the pixel mapping process we can simulate several sky models.

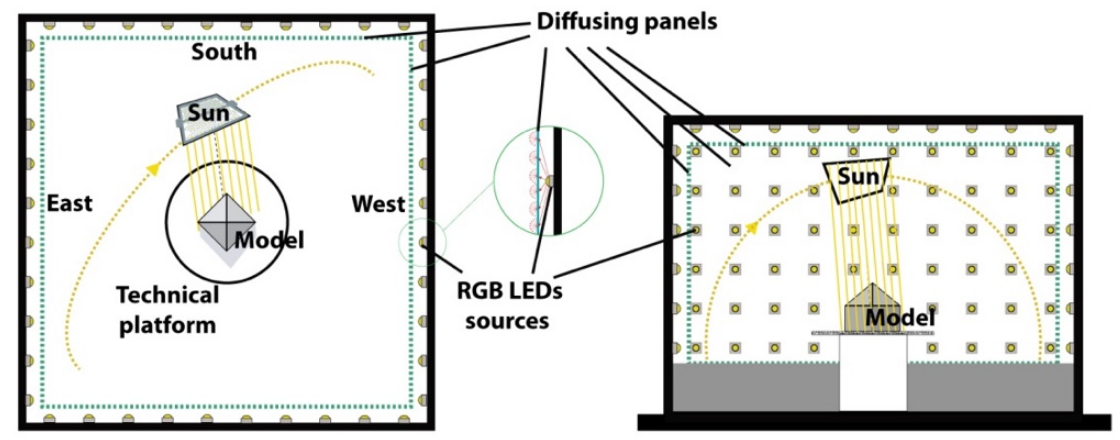

Fig. 12. General principle of the sun \& sky simulator

The entire sun path is reproduced by the mechanical sun moving around the scale model inside the artificial sky. The mechanical sun follows its trajectory on a semi-sphere of a twometer radius. RGBW LED sources which simulate the sun are disposed on a one-meter square plate. The artificial sun allows to study the contribution of light inside the model and its 
shadows. To produce parallel rays, RGBW LED sources are associated with their collimation optics.

\section{Sky models}

The sky models implemented in our software are of two different types: luminance models defined by Perez [11] and the CIE [12] and appearance models defined by Nishita, Haber or Preetham.
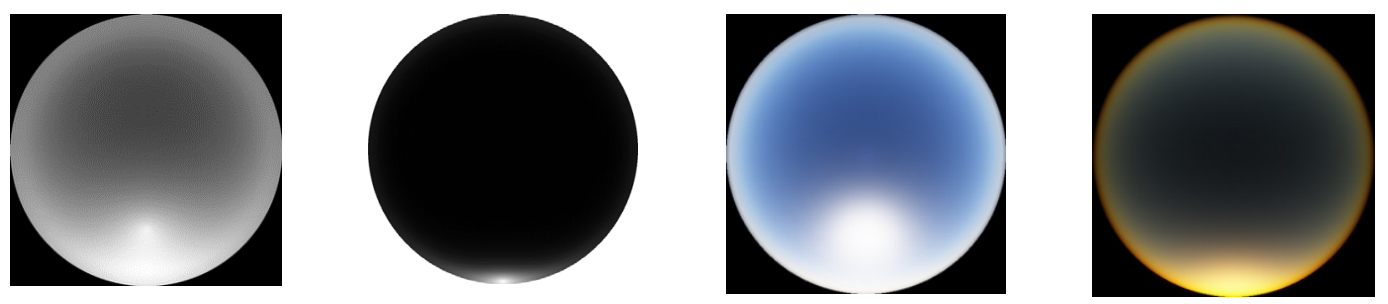

Fig. 13. Overcast sky defined by the CIE at midday, the clear sky sunset defined by Perez, sky image at midday by Haber and sunset image by Nishita.

\subsubsection{Luminance models}

Luminance models can be used to determine the relative luminance of any point in the sky or sky element defined by its height and azimuth. To calculate the luminance of a sky element, three main parameters are considered:

- The sun position which is defined precisely via the Sun Path Algorithm program [13]

- The sky conditions (clear sky, overcast sky...)

- The position of the sky element in the celestial vault

Perez proposes to calculate sky images for 8 ranges of sky clarity from clear to overcast sky. The CIE has standardised equations which define 16 different sky types, including the "standard overcast sky" and the "clear sky with polluted atmosphere".

\subsubsection{Appearance models}

Appearance models are in colour and based on the results of the Perez luminance models. Appearance models appear to be the most interesting to use because the colour increases the realism of the simulation. In order to compare the different sky models an implementation of the main appearance models were made by Bruneton. Its code being freely accessible, modifications were applied to produce the data required for our needs. The models implemented are those of Bruneton, Elek, Haber, Hosek, two models of Nishita (one defined in 1993, and the other in 1996), of O’Neal and Preetham [14-19].

Some are less realistic than others because they have not been defined the same way. For example, some reproduce sunsets better than others. The best models will however be defined during the calibration of the system. 


\subsection{User interface details}

Two computers are used to control and synchronize all the components of the simulator: one sends all the information needed to achieve the experiment, the other one collects the experiment data and gives a visual feedback in real time.

The software handles the computation of the images of the sky, the communication with the Arduino to achieve the pixel mapping, the communication with the motors of the mechanical sun, the DMX communication to control the light sources of the artificial sun, the different video and photo acquisition cameras.

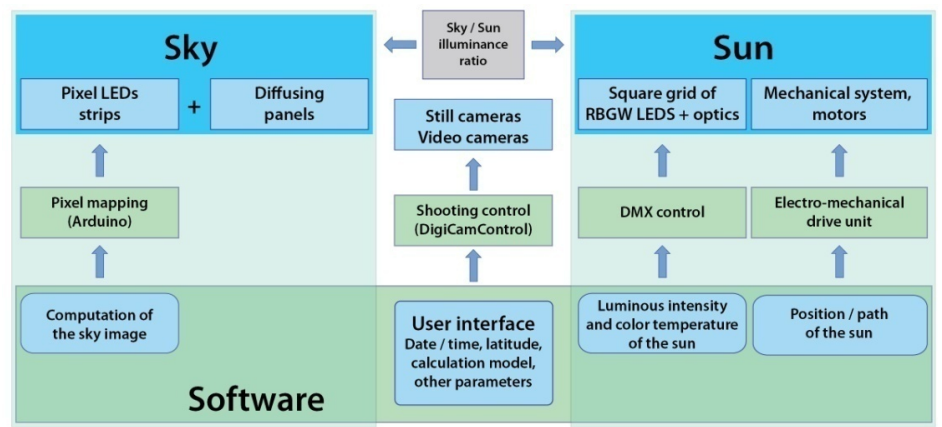

Fig. 14. Software functional chart

The software allows to define the parameters of the experiment, the geographical location, the day, the experiment duration, the sky model, atmosphere conditions (turbidity, albedo), etc.
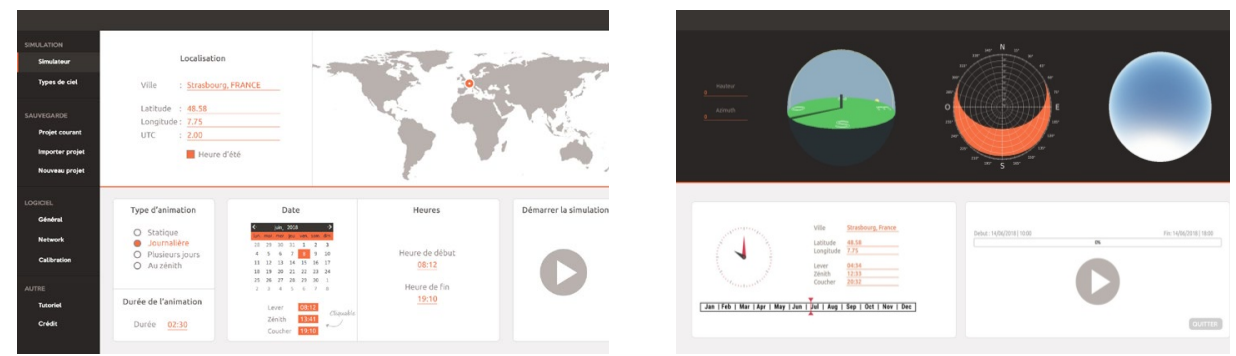

Fig. 15. User interface for simulation settings, solar diagram and other feedback

Once the simulation is started, the interface shows the evolution of the sun on a solar diagram, the current image of the sky, etc.

\section{Conclusion}

As each laboratory develops its own simulators, a state of the art of daylight simulators led us to define the main features of our systems, considering the use of current and emerging technologies. Several prototypes allowed us to study and validate our technological choices.

In our sun and sky simulator, a mechanical sun is integrated inside the artificial sky, moving around the scale model. LED lighting technology allowed us to produce a nonhomogeneous colour system with calculated images displayed on the walls and the ceiling of 
a cubic shape sky. As each LED source composing the sky is individually controlled via a pixel mapping process, it is possible to display in real-time simulations using luminance or appearance sky models. High-power RGBW LED sources associated with their collimation optics on one-meter square aluminium reproduce the sun carried by the mechanics. A sun \& sky simulator is considered as a qualitative device. As we master the pixel mapping software, the calibration processs, sky models, and the visual feedback, our simulator can evolve into a quantitative tool.

In a next step, in-situ photometric measurements, daylight computed simulations and measurements under our simulators will be compared to identify the interest and the limits of each method to produce the most relevant results.

\section{References}

1. Dominique Laburte, Jacques Rizzotti. Camera obscura, sensing spaces - Pour un enseignement expérimental des ambiances. Thibaud, Jean-Paul and Siret, Daniel. Ambiances in action / Ambiances en acte(s) - International Congress on Ambiances, Montreal, Canada. International Ambiances Network, pp.589-594, (2012).

2. D. Laburte, J. Rizzotti, S. Varano, JP. Wetzel De Lumine 7 ENSAS Evaluation des performances de la lumière du jour dans les musées. Rapport de synthèse (20132014)

3. M. Bodart, A. Deneyer. A guide for the building of daylight scale models. In PLEA2006 - The 23rd Conference on Passive and Low Energy Architecture, Geneva, Switzerland, 6-8 September (2006)

4. D. Croghan, Daylighting by Design: 2. Some early examples of the use of an artificial sky, (2015) (unpublished paper, retrieved on ResearchGate)

5. Nawab, M. (1981). DEVELOPMENT AND USE OF A HEMISPHERICAL SKY SIMULATOR. Lawrence Berkeley National Laboratory. Report \#: LBL-13400.

6. Okado, Masaaki \& Nakamura, Hiroshi \& Koga, Yasuko \& Goto, Koichi \& Fujii, Shun'ichi. (1997). Development of an Artificial Sky: part 1: Various Artificial Skies in the World. Journal of the Illuminating Engineering Institute of Japan.

7. Mehrnoush, L.,Prohasky, D.,Burry, J.,Akbarzadeh, A.,Williams, N. (2014). ROBOTHERMODON: An artificial sun study lab with a robot arm and advanced model platform -A thermal heliodon (STEVE: Solar Thermal Evaluation Experiment) In: Proceedings of the 34th Annual Conference of the Association for Computer Aided Design in Architecture (ACADIA) 2014, Los Angeles, United States, 23-25 October (2014)

8. Performance of a new sky scanning simulator / Michel L., Roecker C., Scartezzini, J.-L. - In Lighting Research and Technology, vol. 27, num. 4 (1995), p. 197-208

9. Designing and building a geodesic dome as a bearing structure for an 'artificial sky' lighting installation Maria K. VRONTISSI Proceedings of the International Association for Shell and Spatial Structures (IASS) Symposium 2009, Valencia

10. Richard Kittler, Stanislav Darula, Determination of time and sun position system, Solar Energy, Volume 93, 2013, Pages 72-79, ISSN 0038-092X

11. Perez, R., Seals, R., \& Michalsky, J. (1993). All-weather model for sky luminance distribution-preliminary configuration and validation. Solar energy, 50(3), 235245 . 
12. Darula, S., \& Kittler, R. (2002). CIE general sky standard defining luminance distributions. Proceedings eSim, 11-13.

13. Reda, I.; Andreas, A. (2003). Solar Position Algorithm for Solar Radiation Applications. 55 pp.; NREL Report No. TP-560-34302, Revised January 2008.

14. Nishita, T., Sirai, T., Tadamura, K., \& Nakamae, E. (1993, September). Display of the earth taking into account atmospheric scattering. In Proceedings of the 20th annual conference on Computer graphics and interactive techniques (pp. 175-182). ACM.

15. Nishita, T., Dobashi, Y., Kaneda, K., \& Yamashita, H. (1996, August). Display method of the sky color taking into account multiple scattering. In Pacific Graphics (Vol. 96, pp. 117-132).

16. Preetham, A. J., Shirley, P., \& Smits, B. (1999, July). A practical analytic model for daylight. In Proceedings of the 26th annual conference on Computer graphics and interactive techniques (pp. 91-100). ACM Press/Addison-Wesley Publishing Co..

17. Bruneton, E., \& Neyret, F. (2008, June). Precomputed atmospheric scattering. In Computer Graphics Forum (Vol. 27, No. 4, pp. 1079-1086). Blackwell Publishing Ltd.

18. Elek, O., \& Kmoch, P. (2010, May). Real-time spectral scattering in large-scale natural participating media. In Proceedings of the 26th Spring Conference on Computer Graphics (pp. 77-84). ACM.

19. Hosek, L., \& Wilkie, A. (2012). An analytic model for full spectral sky-dome radiance. ACM Transactions on Graphics (TOG), 31(4), 95. 